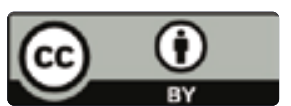

\title{
SARCOPENIA Y FRAGILIDAD COMO PREDICTORES DE RIESGO DE MUERTE EN ADULTOS MAYORES CON ENFERMEDAD RENAL CRONICA EN HEMODIALISIS
}

\section{SARCOPENIA AND FRAGILITY AS PREDICTORS OF RISK OF DEATH IN OLDER ADULTS WITH CHRONIC KIDNEY DISEASE ON HEMODIALYSIS}

\author{
1. Hospital Regional, Ica. \\ 2. Natural and Social Sciences Research \\ a. Médico Internista \\ b. Licenciada en Enfermería
}

Luis Enrique Rojas-Cáceres ${ }^{1, a}$, Melisa Pamela Quispe-Ilanzo ${ }^{2, b}$.

DOI: https://doi.org/10.35563/rmp.v9i1.297

Correspondencia:

M.C. Luis Enrique Rojas Cáceres Dirección:

Número de celular:

Correo Electrónico:

lerc_9@hotmail.com

\section{Contribuciones De Autoría:}

LERC y MPQI participaron en la concepción y diseño del manuscrito, recolección, análisis e interpretación de los datos, redacción y revisión crítica del contenido del manuscrito y aprobación final del artículo.

Conflicto De Intereses: Los autores declaran no tener conflictos de interé Financiamiento: Autofinanciado.

\section{Como Citar}

Rojas Cáceres LE. Quispe Ilanzo MP. Sarcopenia y fragilidad como predictores de riesgo de muerte en adultos mayores con enfermedad renal cronica en hemodialisis. Rev méd panacea. 2020; 9(1): 50-56. doi: https://doi.org/10.35563/rmp.v9i1.297

Recibido: $11-01-2020$

Aceptado: 16 - $02-2020$

Publicado: 06 - 03 - 2020

\section{RESUMEN}

Introducción: Fragilidad y sarcopenia son variables que podrían influir decisivamente en el exitus del paciente con enfermedad renal crónica sometido a hemodiálisis. Objetivo: Determinar el riesgo de muerte de pacientes adultos mayores con enfermedad renal crónica en hemodiálisis que cursan con sarcopenia y fragilidad. Materiales y métodos: Se realizó una búsqueda sistemática de investigaciones publicadas entre los años del 2013 al 2018, en las bases de datos de acceso abierto especializadas en ciencias de la salud: Google Académico, Ebsco, PubMed, Redalyc, Scielo y Lilacs. Resultados: Luego de la búsqueda realizada, se recopilaron nueve estudios internacionales y un estudio nacional. No se ubicaron estudios locales. Conclusión: Sarcopenia y fragilidad se asocian con la peor condición nutricional y clínica, así como con el peor pronóstico en el paciente adulto mayor sometido a hemodiálisis.

Palabras clave: sacopenia - fragilidad - adulto mayor - hemodiálisis - riesgo.

\section{ABSTRACT}

Introduction: Fragility and sarcopenia could decisively influence the exitus of patients with chronic kidney disease undergoing hemodialysis. Objective: To determine the risk of death of elderly patients with chronic kidney disease on hemodialysis who present with sarcopenia and frailty. Materials and methods: A systematic search of research published between 2013 and 2018 was carried out in open access databases specializing in health sciences: Google Scholar, Ebsco, PubMed, Redalyc, Scielo and Lilacs. Results: After the search, nine international studies and one national study were compiled. No local studies were located. Conclusions: Sarcopenia and fragility are associated with a worse nutritional and clinical condition, as well as a worse prognosis in the elderly patient undergoing hemodialysis.

Keywords: sacopenia - frailty - elderly - hemodialysis - risk. 


\section{INTRODUCCIÓN}

En la actualidad existen nuevos desafíos aspectos para los sistemas de salud a nivel mundial, debido al aumento de la esperanza de vida y del envejecimiento progresivo de la población, principalmente en el aspecto cardiovascular y renal ${ }^{1}$. Este aumento en la esperanza de vida, de las últimas décadas, se ha acompañado con el rápido envejecimiento de la población y la mayor prevalencia de enfermedades crónicas; además de, traer como consecuencia la elevada prevalencia de enfermedad renal

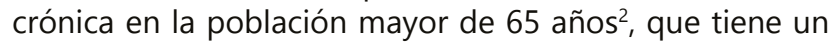
fuerte impacto como causa de muerte en la población mundial ${ }^{3}$ provocando impacto económico, ético, social, política en el individuo, la familia, la comunidad y los servicios de salud ${ }^{4}$. Este envejecimiento de la población y la mejora de la atención médica, ha incrementado el número de pacientes adultos mayores con enfermedad renal en etapa terminal que se vuelven dependientes de la diálisis 5 .

La hemodiálisis es la terapia sustitutiva renal más usada en los pacientes con enfermedad renal crónica en estadio $\mathrm{V}^{7}$ y cuyo éxito necesita de una frecuencia y una duración adecuada $^{8}$. Sin embargo, en los pacientes sometidos a esta terapia, se observan elevadas tasas de mortalidad ${ }^{9}$; en ellos el riesgo estimado de mortalidad por eventos cardiovasculares es más de 10 veces el riesgo de los individuos de la misma edad en la población general ${ }^{10}$. De esta forma se convierte en un problema socioeconómico y de salud pública para todos los sistemas de salud en el mundo que está en aumento. Ocasiona elevada morbimortalidad, incapacidad laboral y aumento de costos sanitarios por el acelerado aumento de pacientes en diálisis, especialmente por el mayor número de adultos mayores $^{2}$.

Al igual que en otros grupos de edad, la insuficiencia renal dependiente de la diálisis ha aumentado entre las personas de edad avanzada durante la última década, impactando así, en el aumento de la tasa de supervivencia y esperanza de vida estos, pero implica numerosos desafíos para ellos porque se encuentran entre los grupos vulnerables y se espera que se exacerbe en la adultez mayor $^{11}$.

A su vez, los pacientes con enfermedades crónicas de larga data y las personas adultas mayores requieren mayor atención por parte de equipos multiprofesionales y cuidadores, debido a que comúnmente sufren de pérdida de independencia, ausentismo en el trabajo, socialización deficiente, empobrecimiento debido a la reducción de los ingresos y aumento de los gastos en salud $y$ reestructuración familiar. La enfermedad renal crónica por lo general tiene un progreso más lento en pacientes adultos mayores. Por lo tanto, pueden permanecer por períodos más largos bajo un tratamiento conservador; sin embargo, la esperanza de vida y la calidad de vida de este grupo tiende a deteriorarse ${ }^{12}$.
El pronóstico es malo en algunos de estos pacientes después de iniciar la diálisis, la mortalidad es alta y tienen un alto riesgo de deterioro funcional ${ }^{5,6}$. A medida que la población en diálisis envejece, sus limitaciones para realizar las actividades diarias afectan el bienestar de los pacientes y aumentan la carga para los cuidadores y el uso de los servicios de salud ${ }^{13}$.

Recientemente, el concepto de fragilidad, el estado de baja reserva homeostática que conduce a una alta vulnerabilidad por cambios repentinos de salud adversos, surgió como un posible buen predictor del pronóstico en la población con enfermedad renal en etapa terminal ${ }^{14}$, debido a que se asocia con un mayor riesgo de resultados adversos, como disminución de la capacidad funcional, aumento de caídas, delirio, institucionalización, hospitalización y muerte. Es un trastorno relacionado con la edad y los cambios en múltiples funciones biológicas, incluido el estado nutricional, la capacidad física, la movilidad, la actividad social, la función cognitiva y el aspecto psicológico ${ }^{15}$.

De igual forma, la sarcopenia es altamente prevalente en el adulto mayor y aparece también en pacientes con enfermedades crónicas. Sin embargo, en la insuficiencia renal crónica contribuyen a su aparición la enfermedad crónica per se, la edad avanzada, el sedentarismo habitual, sumado a múltiples factores que deterioran el estado nutricional, tales como reducción de la ingesta asociada o no a drogas anorexígenas, inflamación crónica, déficit de hormonas anabólicas, bajos niveles de vitamina $D$, resistencia insulínica y disminución de gelsolina (proteína clave en el ensamblaje y desensamblaje de filamentos de actina) ${ }^{16}$.

En ese sentido, estas dos entidades -fragilidad y sarcopenia- podrían influir decisivamente en el exitus del paciente con enfermedad renal crónica sometido a hemodiálisis. Por esta razón se realizó la presente búsqueda sistemática de artículos con el objetivo de determinar el riesgo de muerte de pacientes adultos mayores con enfermedad renal crónica en hemodiálisis que cursan con sarcopenia y fragilidad.

\section{MATERIALES Y MÉTODOS}

Se realizó una búsqueda sistemática de investigaciones publicadas entre los años 2013 y 2018, en las bases de datos de acceso abierto especializadas en ciencias de la salud: Google Académico, Ebsco, PubMed, Redalyc, Scielo y Lilacs, utilizando como términos de búsqueda, en inglés y español, "sarcopenia", "fragilidad", "riesgo", "mortalidad" "diálisis" y "adulto mayor". Se omitieron las secciones "Cartas al editor" y "Editorial".

\section{RESULTADOS.}

Luego de la búsqueda realizada, se recopilaron nueve estudios internacionales. No se ubicaron estudios nacionales ni locales. 
Ziolkowski y col. (Estados Unidos, 2019) ${ }^{17}$, realizaron el studio "Relative sarcopenia and mortality and the modifying effects of chronic kidney disease and adiposity" determinaron las asociaciones de sarcopenia y sarcopenia relativa con la mortalidad, independientemente de las comorbilidades, y si la enfermedad renal crónica y la adiposidad alteran estas asociaciones. Se evaluaron 14 850 participantes de la National Health and Nutrition Examination Survey de 1999 a 2006 y se vincularon datos de certificados de defunción en el National Death Index con seguimiento hasta el año 2011. Se observó que 3\% de los participantes cumplieron con los criterios de sarcopenia y el $8,7 \%$ con los criterios de sarcopenia relativa. La sarcopenia y la sarcopenia relativa se asociaron de forma independiente con la mortalidad (RR sarcopenia $=2,20 ; \quad I C 95 \%=1,69-2,86 ; \quad$ RR sarcopenia relativa $=1,60 ; \quad I C 95 \%=1,31-1,96)$. Los riesgos atribuibles de la población correspondiente fueron 5,2\% (IC95\%=3,4\%-6,4\%) y $8,4 \% \quad$ (IC95\%=4,8\%-11,2\%), respectivamente. La sarcopenia relativa se mantuvo significativamente asociada con la mortalidad $(R R=1,32$; IC95\%=1,08-1,61) cuando se limitó al subconjunto que no cumplía con los criterios de sarcopenia. El riesgo de mortalidad asociado con la sarcopenia relativa se atenuó entre las personas con mayor índice de masa corporal $(p<0,01)$ y no se vio afectado por el estado de la enfermedad renal crónica ni por sarcopenia ni por la sarcopenia relativa. Los autores concluyen que la sarcopenia y la sarcopenia relativa se asocian significativamente con la mortalidad independientemente del estado de la enfermedad renal crónica. La sarcopenia relativa es casi tres veces más frecuente, lo que amplifica el riesgo de mortalidad asociado a nivel de la población. La asociación entre la sarcopenia relativa y la mortalidad se atenúa en las personas con mayor índice de masa corporal.

Kamijo y col. (Japón, 2018) ${ }^{15}$, en su estudio transversal y longitudinal "Sarcopenia and frailty in PD: impact on mortality, malnutrition, and inflammation", evaluaron la morbilidad y la mortalidad de la sarcopenia y la fragilidad en pacientes con diálisis peritoneal. Incluyeron a 119 pacientes en quienes se observó que la presencia de sarcopenia y fragilidad fue $8,4 \%$ y $10,9 \%$, respectivamente. Asimismo, durante el seguimiento, la presencia de sarcopenia o fragilidad se asoció con el riesgo de mortalidad. En el análisis multivariado, la fragilidad se relacionó con la tasa de mortalidad de los pacientes con diálisis peritoineal. Asimismo, la presencia de sarcopenia o fragilidad se asociaron con un peor pronóstico.

Gilgio y col. $\left(\right.$ Brasil, 2018) ${ }^{18}$, desarrollaron el estudio "Association of sarcopenia with nutritional parameters, quality of life, hospitalization, and mortality rates of elderly patients on hemodialysis" evaluaron si la disminución de la masa muscular, la disminución de la fuerza muscular o ambas afecciones (sarcopenia) se asocian con un peor estado nutricional, mala calidad de vida (CV) y resultados difíciles, como la hospitalización y la mortalidad, en pacientes adultos mayores sometidos a hemodiálisis. Diseñaron un estudio multicéntrico de observación longitudinal que incluyó a 170 pacientes hemodiálisis (edad $70 \pm 7$ años, $65 \%$ hombres) de 6 centros de diálisis. En ellos se observó una masa muscular reducida en el $64 \%$ de los pacientes, una fuerza muscular reducida en el $52 \%$ y sarcopenia en el $37 \%$. El grupo con sarcopenia era mayor, tenía una mayor proporción de hombres y mostró peores condiciones clínicas y nutricionales en comparación con los pacientes sin sarcopenia. Aunque la reducción de la masa muscular estuvo fuertemente asociada con un mal estado nutricional, la baja fuerza muscular se asoció con peores dominios de la calidad de vida. En los análisis multivariantes de Cox ajustados por edad, sexo, edad de diálisis y diabetes mellitus, la fuerza muscular baja sola y la sarcopenia se asociaron con una mayor hospitalización, mientras que la sarcopenia fue un predictor de mortalidad. En conclusión, a sarcopenia se asoció con peores condiciones nutricionales y clínicas y fue un predictor de hospitalización y mortalidad.

Androga y col. (Estados Unidos, 2017) ${ }^{19}$, en su estudio "Sarcopenia, obesity, and mortality in US adults with and without chronic kidney disease", determinaron si las asociaciones de composición corporal con la mortalidad por todas las causas difieren entre los portadores de enfermedad renal crónica en comparación con aquellos que no cursan con la enfermedad. Examinaron adultos estadounidenses 20 o más años $(n=15$ 332) del National Health and Nutrition Examination Survey (NHANES) que contaban con datos de composición corporal disponibles $(n=12732)$, excluyendo participantes con tasa de filtrado glomerular estimado $<15 \mathrm{ml} / \mathrm{min} / 1,73 \mathrm{~m} 2(n=34) 0$ datos faltantes en las covariables de interés ( $n=1082)$. La cohorte resultante tuvo 11616 participantes. Los resultados demostraron que la enfermedad renal crónica modificó la asociación de la composición corporal con la mortalidad ( $p=0,01$ para la interacción). En los participantes sin enfermedad, tanto la sarcopenia como la obesidad sarcopénica se asociaron de forma independiente con el aumento de la mortalidad en comparación con la composición corporal normal $(R R=1,44 ; I C 95 \%=1,07-1,93 ;$ y $R R=1,64 ; I C 95 \%=1,26-2,13$, respectivamente). Estas asociaciones no estuvieron presentes entre los participantes con enfermedad renal crónica. Por el contrario, las personas obesas tenían el menor riesgo ajustado de muerte, con un mayor riesgo entre las personas con sarcopenia $(R R=1,43$; IC95\% $=1,05-1,95)$ pero no sarcopenia-obesidad $(p=0,003$ para la interacción por estado de enfermedad renal crónica; $R R=1,21$; IC95\%=0,89-1,65), en comparación con la obesidad. Los autores concluyen que la sarcopenia se asocia con una mayor mortalidad independientemente de la tasa de filtración glomerular estimada, pero el exceso de adiposidad modifica esta asociación entre las personas con enfermedad renal crónica.

Lee y col. (Korea del Sur, 2017) ${ }^{20}$, en su estudio prospectivo "The prevalence, association, and clinical outcomes of frailty in maintenance dialysis patients" 
investigaron las implicaciones clínicas de la fragilidad en pacientes con enfermedad renal crónica en hemodiálisis de mantenimiento y diálisis peritoneal crónica. Se reclutaron 1658 pacientes (1 255 hemodiálisis de mantenimiento y 403 diálisis peritoneal crónica) de 27 unidades de multidiálisis. La edad promedio de los participantes fue de 55,2 \pm 11,9 años y el 55,2\% eran hombres. Entre los participantes, el 34,8\% fue calificado como frágil y el $45,7 \%$ como prefrágil. Durante el período de seguimiento (mediana de 17,1 meses), 608 pacientes (79 no frágiles, 250 prefileados y 279 frágiles) fueron hospitalizados, y 87 pacientes (10 no frágiles, 24 prefabricados y 53 frágiles) murieron $(p<0,001)$. La fragilidad se asoció con la hospitalización ( $R R=1,80$; IC95\%: 1,38-2,36) y mortalidad $(R R=2,37$, IC95\%=1,11-5,02). Los autores concluyen que el fenotipo de fragilidad fue común incluso en pacientes con diálisis en etapa terminal prevalentes en diálisis, y se asoció significativamente con mayores tasas de hospitalización y mortalidad.

Rena y col. (China, 2016) ${ }^{21}$, en su estudio descriptivo "Sarcopenia in patients undergoing maintenance hemodialysis: incidence rate, risk factors and its effect on survival risk" investigaron la incidencia de sarcopenia en pacientes con hemodiálisis de mantenimientos y sus factores influyentes, así como su impacto en el riesgo de supervivencia evaluó a 131 pacientes. La incidencia de sarcopenia en toda la muestra fue $13,7 \%$ y la incidencia de sarcopenia en pacientes mayores de 60 años 33,3\%. El análisis de supervivencia de Kaplan-Meier mostró una supervivencia a un año del $88,9 \%$ en los pacientes sarcopénicos, que fue significativamente menor que en los pacientes no sarcopénicos. En conclusión a un año de seguimiento se encontró que el riesgo de mortalidad de los pacientes sarcopénicos era mayor que el de los pacientes no sarcopénicos.

Pugh y col. (Reino Unido, 2016)22, en su investigación observacional de seguimiento "Frailty and comorbidity are independent predictors of outcome in patients referred for pre-dialysis education" evaluaron el impacto de la comorbilidad y la fragilidad en los resultados de los pacientes remitidos para educación previa a la diálisis evaluó a 283 pacientes remitidos para educación previa a la diálisis. Entre los resultados observaron que las puntuaciones de comorbilidad y fragilidad clínica en el momento de la derivación al servicio de prediálisis fueron factores predictivos independientes de mortalidad. Dentro del período de seguimiento del estudio, el $76 \%$ de los pacientes con un alto puntaje de fragilidad clínica en el momento de la educación previa a la diálisis había fallecido, y el $63 \%$ de estos pacientes no comenzaron la diálisis antes de la muerte.

Alfaadhel y col. (Canadá, 2015) ${ }^{23}$, en su estudio observacional de cohorte "Frailty and mortality in dialysis: evaluation of a clinical frailty scale" evaluaron si hubo una asociación entre el grado de fragilidad y la mortalidad entre los pacientes en diálisis. La cohorte consistió en 390 pacientes con evaluación completa de fragilidad clínica (edad promedio de $63 \pm 15$ años). La mayoría eran caucásicos (89\%) y hombres (67\%). El 30\% de los pacientes tenían insuficiencia renal crónica terminal causada por nefropatía diabética. La mediana de la puntuación del índice de comorbilidad de Charlson fue de 4 (rango intercuartil = 3-6), y la mediana de la puntuación de fragilidad clínica fue de 4 (rango intercuartil = 2-5). Hubo 96 muertes en 750 pacientes-años en riesgo. En un análisis de supervivencia de Cox ajustado, el índice de riesgo asociado con cada aumento de 1 punto en el inventario de fragilidad clínica fue de 1,22 (IC95\%=1,04-1,43; $p=0,02)$. Los autores concluyen que la mayor severidad de la fragilidad en el inicio de la diálisis se asocia con una mayor mortalidad.

McAdams-DeMarco y col. (Estados Unidos, 2013) ${ }^{24}$, en su estudio prospectivo de cohorte "Frailty as a novel predictor of mortality and hospitalization in hemodialysis patients of all ages" cuantificaron la prevalencia de la fragilidad en pacientes adultos de todas las edades sometidos a hemodiálisis crónica, su relación con la comorbilidad y la discapacidad, y su asociación con resultados adversos de mortalidad y hospitalización. Evaluaron a 146 pacientes con hemodiálisis. Al inicio del estudio el $50 \%$ de los pacientes de hemodiálisis mayores (edad $\geq 65$ ) y el $35,4 \%$ de los pacientes más jóvenes (edad $<65)$ eran frágiles; el 35,9\% y el 29,3\% fueron frágiles intermedios, respectivamente. La mortalidad a los tres años fue $16,2 \%$ para los no frágiles, 34,4\% para los frágiles intermedios y $40,2 \%$ para los frágiles. La fragilidad intermedia y la fragilidad se asociaron con un riesgo de muerte mayor de 2,68 veces (IC 95\% $=1,02-7,07, p=0,046$ ) y 2,60 veces (IC95\%: 1,04-6,49, $p=0,041$ ), respectivamente, independientemente de la edad, el sexo, la comorbilidad y la discapacidad. En el año posterior al inicio del estudio, la mediana del número de hospitalizaciones fue de uno (IQR 0-3). La proporción con dos o más hospitalizaciones fue $28,2 \%$ para los no frágiles, $25,5 \%$ para los frágiles intermedios y $42,6 \%$ para los frágiles. La fragilidad intermedia no se asoció con el número de hospitalizaciones ( $R R=0,76$, IC95\%: 0,49-1,16, $p=0,21$ ), la fragilidad se asoció con 1,43 veces (IC95\%=1,00-2,03, $\mathrm{p}=0,049$ ) mayor número de hospitalizaciones independientes de la edad, el sexo, la comorbilidad y la discapacidad. La asociación de la fragilidad con la mortalidad y las hospitalizaciones no difirió entre los participantes mayores y los más jóvenes (Interacción $p=0,64$ y $p=0,14$, respectivamente). Los autores concluyen que los adultos de todas las edades sometidos a hemodiálisis tienen una prevalencia muy alta de fragilidad, más de cinco veces mayor que los adultos mayores. Asimismo, en esta población, independientemente de la edad, la fragilidad es un predictor fuerte e independiente de la mortalidad y el número de hospitalizaciones.

\section{DISCUSIÓN.}

La enfermedad renal crónica generalmente progresa más 
lentamente en los adultos mayores, por lo tanto, pueden permanecer por períodos más largos bajo un manejo conservador; sin embargo, la esperanza de vida y la calidad de vida tienden a deteriorarse en estas personas cuando son sometidos a diálisis. Además, existe controversia sobre los beneficios de la diálisis y cuándo iniciarla o detenerla. Los primeros tres meses de diálisis son críticos, ya que los pacientes experimentan una disminución en la capacidad funcional y la calidad de $v \operatorname{vida}^{25}$. La esperanza de vida disminuye, aproximadamente un tercio no sobrevive después de un año de diálisis y sólo la quinta parte está vivo después de cinco años de tratamiento, con una esperanza de sobrevivencia de ocho meses en aquellos que tienen 90 años a más ${ }^{26}$.

A medida que los pacientes de diálisis envejecen, las complicaciones derivadas de los trastornos nutricionales se vuelven más comunes, con una prevalencia reportada de 30 a $60 \%$, y son más graves en pacientes mayores. Varias patologías relacionadas con la desnutrición pueden actuar como factores que afectan profundamente el pronóstico y la calidad de vida; por lo tanto, tales patologías representan problemas importantes que deben abordarse en relación con el envejecimiento de los pacientes con enfermedad renal crónica y los pacientes con diálisis ${ }^{15}$.

Existen varios factores relacionados que incluyen: disminución de la ingesta dietética, acumulación de sustancias urémicas, efectos catabólicos de la terapia de reemplazo renal, estrés oxidativo, desequilibrios metabólicos y hormonales, aumento de la resistencia a la insulina, inflamación sistémica y afecciones comórbidas. Este complejo conjunto de factores conduce a una disminución en las fuentes de energía y masa muscular, que se conceptualiza como sarcopenia, fragilidad, estados de reducción de masa muscular y deterioro físico, social y mental. También se sabe que estas condiciones se correlacionan entre sí como un concepto de enfermedad relacionado con la desnutrición ${ }^{15,27}$.

La sarcopenia o pérdida de masa y función musculares, no ocurre solamente por disminución del tamaño sino también del número de fibras musculares. En el adulto mayor es un proceso lento, calculándose que la pérdida de la masa muscular comienza alrededor de los 30 años de edad, decreciendo desde entonces y hasta los 80 años alrededor de un 30\%. La pérdida de fuerza y de masa muscular es mayor en los músculos de los miembros inferiores, comparados con los superiores, aun luego de ajustar por la magnitud de las actividades diarias, lo que podría deberse a que el envejecimiento compromete selectivamente más la masa y la función de los músculos de los miembros inferiores, o bien a que estos últimos se utilizan menos en las actividades diarias. Su presencia correlaciona con mayor mortalidad, discapacidad y aumento del riesgo de caídas ${ }^{16}$.
En los últimos años el concepto de fragilidad como «estado de prediscapacidad» se ha extendido de forma amplia en todos los que trabajamos en beneficio de la persona mayor, pero no existe consenso sobre la definición exacta de fragilidad $^{28}$ y existen diferentes instrumentos para evaluarla ${ }^{29}$. Sin embargo, puede considerarse como un estado de baja reserva homeostática (disminución de la reserva fisiológica y desregulación multisistémica) asociada con una mayor vulnerabilidad a los factores estresantes caracterizada por cambios repentinos de salud adversos $14,24,30$. Su importancia radica no solo en su elevada prevalencia - superior al $25 \%$ en mayores de 85 años-, sino a que es considerada un factor de riesgo independiente, que confiere a los adultos mayores que lo presentan un riesgo elevado de discapacidad, institucionalización y mortalidad $^{14,31}$. Expone al doble de riesgo de mortalidad y hospitalización, así como otros resultados adversos, incluyendo caídas, disminución de la movilidad, limitaciones físicas, deterioro respiratorio y deterioro cognitivo $^{24,32}$. Además, es predictiva de retraso en la función del injerto ${ }^{33}$, reingreso hospitalario temprano ${ }^{34}$ y mortalidad $^{35}$ después del trasplante de riñón. Los pacientes frágiles sometidos a hemodiálisis presentan un riesgo de mortalidad 2,6 veces mayor y un riesgo de hospitalización 1,4 veces mayor, independientemente de la edad, el sexo, la comorbilidad y la discapacidad, en comparación con los pacientes que no eran frágiles ${ }^{14}$.

La sarcopenia se enfoca en el deterioro de las funciones físicas que acompañan al envejecimiento, especialmente en diálisis, la fragilidad se enfoca más en los aspectos funcionales de la vida social, la función cognitiva, las actividades de la vida diaria y la función física ${ }^{15}$.

\section{CONCLUSIÓN}

Sarcopenia y fragilidad se asocian con la peor condición nutricional y clínica, así como con peor pronóstico en el paciente adulto mayor sometido a hemodiálisis. 


\section{REFERENCIAS BIBLIOGRÁFICAS}

1. Organización Mundial de la Salud. Estadísticas Sanitarias Mundiales (internet). 2014 (citado 13 oct 2016). Disponible en:

http://www.who.int/research/es/

2. Calderón CA, Urrego JC. Diálisis en el adulto mayor: Mortalidad, calidad de vida y complicaciones. Acta Med Colomb. 2014; 39: 359-367.

3. GBD 2013 Mortality and Causes of Death Collaborators. Global, regional, and national age-sex specific all-cause and cause-specific mortality for 240 causes of death, 1990-2013: a systematic analysis for the Global Burden of Disease Study 2013. Lancet. 2015;385(9963):117-71.

4. Martínez A, Frómeta A, Boza PE. Supervivencia del adulto mayor diagnosticado con enfermedad renal crónica terminal en tratamiento de hemodiálisis. Multimed 2014; 18(3):e1-e27.

5. Collins AJ, Foley RN, Herzog C, et al. US renal data system 2012 annual data report. Am J Kidney Dis. 2013; 61(A7):e1-e476.

6. Drost D, Kalf A, Vogtlander N, van Munster BC. High prevalence of frailty in end-stage renal disease. Int Urol Nephrol. 2016; 48:1357-1362.

7. Karopadi AN, Mason G, Rettore E, Ronco C. Cost of peritoneal dialysis and haemodialysis across the world. Nephrol Dial Transplant. 2013; 28(10): 2553-69.

8. Herrera-Añazco $P, \quad$ Palacios-Guillen $M$, Mezones-Holguin $\quad E$ Hernández AV, Chipayo-Gonzales D. Baja adherencia al régimen de hemodiálisis en pacientes con enfermedad crónica renal en un hospital de referencia del Ministerio de Salud en Perú. An Fac med. 2014; 75(4):323-6.

9. Kalousová $M$, Benáková $H$, Kuběna $A A$, Dusilová-Sulková S, Tesař V, Zima $T$. Pregnancy-Associated Plasma Protein $A$ as an Independent Mortality Predictor in Long-Term Hemodialysis Patients. Kidney Blood Press Res. 2012; 35:192-201.

10. Abdelmalek JA, Stark $P$, Walther $C P$, Ix JH, Rifkin DE. Associations between coronary calcification on chest radiographs and mortality in hemodialysis patients. Am J kidney Dis J Nat Kidney Foundation. 2012; 60(6):990-997.

11. Sahaf $R$, Ilali ES, Peyrovi $H$, Akbari Kamrani AA, Spahbodi F. Uncertainty, the overbearing lived experience of the elderly people undergoing hemodialysis: a qualitative study. IJCBNM. 2017; 5(1):13-21.

12. Braz. J. Reflections on end-of-life dialysis. Nephrol. (J. Bras. Nefrol.) 2018; 40(3):209-212.

13. Cook WL, Jassal SV. Functional dependencies among the elderly on hemodialysis. Kidney International. 2008; 73:1289-1295.

14. McAdams-DeMarco MA, Law A, Salter ML et al. Frailty as a novel predictor of mortality and hospitalization in individuals of all ages undergoing hemodialysis. J Am Geriatr Soc. 2013; 61:896-901.
15. Kamijo $Y$, Kanda E, Ishibashi $Y$, Yoshida $M$. Sarcopenia and frailty in PD: impact on mortality, malnutrition, and inflammation. Perit Dial Int. 2018; 38(6):447-454.

16. Cusumano AM. Sarcopenia en pacientes con y sin insuficiencia renal crónica: diagnóstico, evaluación y tratamiento. Nefrología, Diálisis y Trasplante. 2015; 35(1):32-43.

17. Ziolkowski SL, Long J, Baker JF, Chertow GM, Leonard MB. Relative sarcopenia and mortality and the modifying effects of chronic kidney disease and adiposity. Journal of Cachexia, Sarcopenia and Muscle. 2019; 10: 338-346.

18. Giglio J, Kamimura M, Lamarca F, Rodrigues J, Santin $F$, Avesani CM. Association of sarcopenia with nutritional parameters, quality of life, hospitalization, and mortality rates of elderly patients on hemodialysis. Journal of Renal Nutrition. 2018, 28(3):197-207.

19. Androga L, Sharma D, Amodu A, Abramowitz MK. Sarcopenia, obesity, and mortality in US adults with and without chronic kidney disease. Kidney Int Rep. 2017; 2:201-211.

20. Lee SY, Yang DH, Hwang E, Kang SH, Park SH, Kim TW, et al. The prevalence, association, and clinical outcomes of frailty in maintenance dialysis patients. J Ren Nutr. 2017; 27(2):106-112.

21. Rena H, Gonga D, Jiaa F, Xua B, Liu Z. Sarcopenia in patients undergoing maintenance hemodialysis: incidence rate, risk factors and its effect on survival risk. Renal Failure. 2016; 38(3):364-371.

22. Pugh J, Aggett J, Goodland A, Prichard A, Thomas N, Donovan $\mathrm{K}$, et al. Frailty and comorbidity are independent predictors of outcome in patients referred for pre-dialysis education. Clinical Kidney Journal. 2016; 9(2):324-329.

23. Alfaadhel TA, Soroka SD, Kiberd BA, Landry $D$, Moorhouse $\mathrm{P}$, Tennankore KK. Frailty and mortality in dialysis: evaluation of a clinical frailty scale. Clin J Am Soc Nephrol. 2015; 10(5):832-840.

24. McAdams-DeMarco MA, Law A, Salter ML, Boyarsky B, Gimenez L, Jaar BG, et al. Frailty as a novel predictor of mortality and hospitalization in hemodialysis patients of all ages. J Am Geriatr Soc. 2013; 61(6): 896-901.

25. Verberne WR, Geers $A B$, Jellema WT, Vincent $H H$, van Delden JJ, Bos WJ. Comparative survival among older adults with advanced kidney disease managed conservatively versus with dialysis. Clin J Am Soc Nephrol 2016; 11633-40.

26. Tzanno-Martins C. Reflections on end-of-life dialysis. Braz. J. Nephrol. (J. Bras. Nefrol.). 2018; 40(3):209-212.

27. Chen LK, Liu LK, Woo J, Assantachai P, Auyeung TW, Bahyah KS, et al. Sarcopenia in Asia: consensus report of the Asian working group for sarcopenia. J Am Med Dir Assoc. 2014; 15:95-101. 


\section{REFERENCIAS BIBLIOGRÁFICAS}

28. Clegg A, Young J, Iliffe $S$, et al. Frailty in elderly people. Lancet. 2013; 381:752-762.

29. Bouillon K, Kivimaki M, Hamer $M$, et al. Measures of frailty in population-based studies: an overview. BMC Geriatr. 2013; 13:64.

30. Salter ML, Gupta N, Massie AB, McAdams-De Marco $M A$, Law $A H$, Jacob $R L$, et al. Perceived frailty and measured frailty among adults undergoing hemodialysis: a cross-sectional analysis. Salter et al. BMC Geriatrics. 2015; 15(52):1-7.

31. Portilla ME. Tornero F, Gi P. La fragilidad en el anciano con enfermedad renal crónica. Nefrologia. 2016;3 6(6):609-615

32. McAdams-DeMarco MA, Suresh $S$, Law A, Salter ML, Gimenez LF, Jaar BG, et al. Frailty and falls among adult patients undergoing chronic hemodialysis: a prospective cohort study. BMC Nephrol. 2013;14:224.

33. Garonzik-Wang JM, Govindan P, Grinnan JW, Liu M, Ali HM, Chakraborty A, et al. Frailty and delayed graft function in kidney transplant recipients. Arch Surg. 2012;147(2):190-3.

34. McAdams-DeMarco MA, Law A, Salter ML, Chow E, Grams M, Walston J, et al. Frailty and early hospital readmission after kidney transplantation. Am J Transplant. 2013;13(8):2091-5.

35. McAdams-Demarco MA, Law A, King E, Orandi B, Salter $\mathrm{ML}$, Alachkar $\mathrm{N}$, et al. Frailty and mortality in kidney transplant recipients. Am J Transplant. 2015;15(1):149-54.

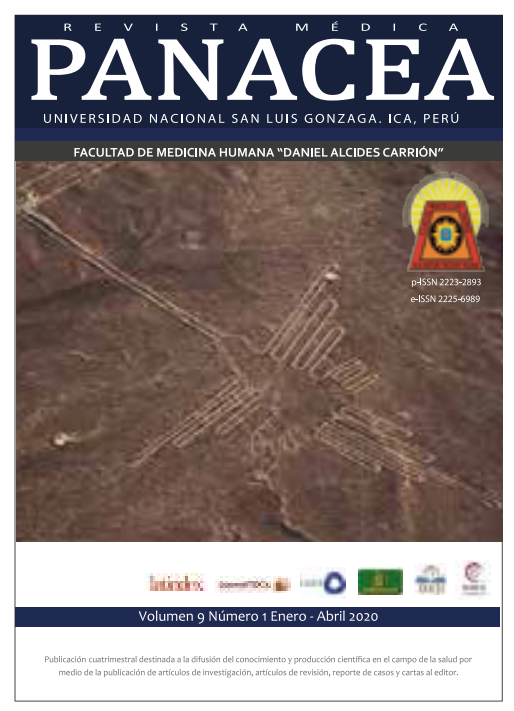

